ANA CRAVINO

FACULTAD DE DISEÑO Y COMUNICACIÓN

UNIVERSIDAD DE PALERMO

BUENOS AIRES, ARGENTINA.

CRAVINO.ANA@GMAIL.COM

JORGE POKROPEK

FACULTAD DE DISEÑO Y COMUNICACIÓN

UNIVERSIDAD DE PALERMO

BUENOS AIRES, ARGENTINA

JORGEPOKROPEK@GMAIL.COM

\section{Racionalidad limitada: las dificultades de la toma de decisiones en el Diseño para la sustentabilidad}

\author{
Bounded rationality: Difficulties to make decisions \\ in Design for sustainability
}

Resumen. El objeto de este artículo es reflexionar sobre los distintos enfoques que implica un diseño ambientalmente consciente. Es sabido que la variabilidad de los datos referidos a la sustentabilidad de un material y la confiabilidad de los mismos constituye un obstáculo para la toma de decisiones. Es por ello que el diseño sustentable, muchas veces, recurre a aspectos formales que ponen en evidencia el reciclaje o reutilización de materiales con una mirada fuertemente artesanal.

Palabras clave: diseño para el medio ambiente, diseño para la sustentabilidad, eco-diseño, racionalidad limitada, reciclaje.

\begin{abstract}
The purpose of this article is to reflect on the different approaches involved in an environmentally conscious design. It is known that the variability of the data referred to the sustainability of a material and the reliability of the same constitutes an obstacle for the decision making. That is why sustainable design, often, resorts to formal aspects that highlight the recycling or reuse of materials with a strongly artisan look.
\end{abstract}

Keywords: bounded rationality, design for the environment, ecodesign, recycling, sustainable design
Financiamiento: Línea de investigación

$\mathrm{N}^{\circ} 3$ financiada por la Facultad de Diseño y

Comunicación, Universidad de Palermo.

Fecha de recepción: 15/06/2018

Fecha de aceptación: 26/09/2018

Cómo citar: Cravino, A. y Pokropek, J. (2018)

Racionalidad limitada: las dificultades

de la toma de decisiones en el Diseño

para la sustentabilidad. RChD: creación y

pensamiento, 3(5), 1-11.

DOI: 10.5354/0719-837X.2018.49742

RChD: creación y pensamiento

Universidad de Chile

2018, 3(5)

http://rchd.uchile.cl 


\section{Introducción}

Esta convocatoria nos invita a analizar un tema que, como diría esa vieja canción de Bob Dylan, está "soplando en el viento": el deterioro creciente y manifiesto de nuestro medio ambiente. El agotamiento de los combustibles fósiles, la contaminación y la degradación de los ecosistemas, junto con la pérdida de diversidad biológica y cultural, ponen en crisis no sólo nuestras ideas, sino también nuestras costumbres y valores, haciendo, de este modo más compleja nuestra toma de decisiones.

Revisando la historia vemos que el paradigma positivista imperante desde el inicio de la modernidad ha insistido en una metodología que tiende a fragmentar los fenómenos y los procesos, para verlos como si se tratara de sistemas cerrados auto-referidos y no de sistemas abiertos, excluyendo la relación e interdependencia entre las distintas estructuras (Capra, 2003). Asimismo, desde la Economía Política se considera que la ausencia de un desarrollo sustentable en los países centrales tiene que ver con un exceso de consumismo manifestado por la irracional sobreexplotación de los recursos, mientras, que en los países periféricos, esta falta de desarrollo se relaciona con la pobreza, y sus consecuencias son la falta de agua y cloacas, la presencia de basurales a cielo abierto, los ríos contaminados, entre otros.(Meadows, 1982) Diversas críticas formuladas ya desde la década del setenta por investigadores de la Fundación Bariloche (Herrera, 1977) y de la CEPAL (CEPAL, 1971) concluyen que el estilo de desarrollo que deberían impulsar los países latinoamericanos tiene que respaldarse, como principio excluyente, en la elevación sustancial de los niveles de vida de su población, considerando la necesidad de realizar "cambios en la estructura de clases y grupos y en la organización social, transformaciones culturales y de valores, cambios en las estructuras políticas y de poder". (Sunkel \& Gligo, 1980, pág. 10) (Giglo, 2006) En esta serie de mutaciones el diseño puede jugar un importante papel, en el sentido de modificar los valores que la sociedad tiene respecto a la sustentabilidad, generando un uso responsable de objetos y servicios.

En América Latina es claro entonces que el logro de la sostenibilidad aparece hoy indisolublemente asociado a la necesidad de universalización y ampliación de los derechos humanos. Recordemos que en la Cumbre para la Tierra de 1992 se reconoció internacionalmente que la protección del medio ambiente y la administración de los recursos naturales deben integrarse en las cuestiones socio-económicas de pobreza y subdesarrollo. También es cierto que los sectores populares, por obvias razones económicas, tienen una tendencia a reutilizar, reciclar y extender la vida útil de los objetos. Sin embargo, este sector de la sociedad no puede acceder tan fácilmente a la administración de recursos naturales, siendo, muchas veces, víctimas de la contaminación y de los efectos del sobreconsumo de terceros, del cual no han obtenido ningún beneficio directo.

Asimismo, el economista bengalí Amartya Sen concibe la mejora de los pueblos como un proceso de expansión de las libertades reales de las que disfrutan los individuos, alejándose de la visión tradicional que asocia el progreso con el simple crecimiento del PBI, el grado de la industrialización, los avances tecnológicos o los ingresos personales. (Vílchez, Gil Pérez, Toscano, \& Macías, 2010) La expansión de las libertades es, para este autor, tanto un fin como un medio para alcanzar el desarrollo y constituye un pilar fundamental para abordar la problemática de la sostenibilidad. Pero estas libertades están acotadas por los márgenes en que tomamos nuestras decisiones, entre ellas, las proyectuales. 
Algunos autores recurren a Guattari para señalar que la ecología medio ambiental no tiene salida sin una "ecología de las ideas" que "debe percibirse en la organización de los sistemas sociales en los que los individuos producen y se producen colectivamente" (Cangi, 2015, pág. 90). Es por ello que el diseño tiene que ser un motor de transformación de dichas ideas.

Sin embargo, para tomar decisiones ambientalmente conscientes hay que contar con datos fehacientes, por ejemplo, respecto al ciclo de vida de los materiales, además de criterios rigurosos referidos al tipo de material adecuado para una determinada situación.

Es, entonces, objeto de este trabajo, analizar, desde una metodología cualitativa, sobre qué base se toman las decisiones de diseño cuando falta información, y qué tipo de acciones se llevan a cabo en esa circunstancia. Este escrito forma parte de la línea de investigación "Forma y Materialidad" que se desarrolla en la Facultad de Diseño y Comunicación de la Universidad de Palermo, Argentina.

\section{Diseño Sustentable. Definiciones}

Es muy fácil llegar a un acuerdo con respecto a lo que entendemos por "Arquitectura Bioclimática" o "Arquitectura Sustentable" y a las posibilidades reales de concretar este tipo de resultado. Son innumerables los manuales (Evans \& De Schiller, 1988) (Olgyay, 1998) (D’Amico, 2000), entre los muchos a los que podemos acceder para obtener fórmulas y procedimientos. $Y$ también hay ya a escala mundial y en nuestro medio, numerosos centros de estudio que empiezan a ocuparse de este tema ${ }^{1}$.

Sin embargo “Diseño sustentable"no es un concepto que se encuentre definido con precisión, más allá de un conjunto de deseos o propósitos, ligados muchas veces a la elección de un material (Iussa, Granero, Govoni, Argüello, \& Gatani, 2015), que permite otorgar la categoría de "sustentable" de manera independiente a las propias acciones de diseño. Por eso el INTI -Instituto Nacional de Tecnología Industrial de la Argentina- afirma que

Fomentamos el diseño para la sustentabilidad como herramienta de innovación; reduciendo los impactos ambientales, obteniendo una mejora en la competitividad de las organizaciones y mejorando la inclusión de los actores sociales. Promovemos el enfoque de Ciclo de Vida como herramienta central de los diseñadores para acercarse a este objetivo. ${ }^{2}$

Respecto a la sustentabilidad señala Marta Astier que:

Hablamos de un «metaconcepto»-tal como democracia o justicia- que parte de principios generales y resulta de aplicación universal. Sin embargo, no existe una definición única de sustentabilidad, pues en última instancia depende del sistema de valores. De hecho existe una multiplicidad de perspectivas válidas para su definición y análisis(...) Por este motivo, la sustentabilidad debe definirse localmente, prestando atención a la diversidad sociocultural y ambiental. Es un concepto complejo y multidimensional que implica entender la interrelación entre aspectos ambientales, económicos y sociales.

(Astier, Masera, \& Galván-Miyoshi, 2008, pág. 42)
1. Instituto de Acondicionamiento Ambiental, Universidad Nacional de Tucumán, Laboratorio de Ambiente Humano y Vivienda, Centro Regional de Investigaciones Científicas y Técnicas, Conicet, Mendoza; Instituto de Investigaciones Tecnológicas para el Diseño Ambiental del Hábitat Humano, Facultad de Arquitectura y Urbanismo (FAU), Universidad Nacional del Nordeste (UNNE); Instituto de Estudios del Hábitat, Línea de Investigación: Hábitat y Energía, Universidad Nacional de la Plata UNLP Inenco (Instituto de Investigación en Energías no Convencionales), UNS, universidad Nacional de Salta 2. https://www.inti.gob.ar/disenoindustrial/ sustentable.htm 
Sabemos que durante la década del 80 y parte de los 90 , la sustentabilidad era un tema referido casi exclusivamente al medio ambiente, aunque luego la cuestión se fue orientando hacia las llamadas "tecnologías limpias" y la "eco-eficiencia". Posteriormente se empezó a evaluar el impacto de la producción tomando en consideración todo el ciclo de vida. Es por ello que conceptos como Diseño para el Ambiente (DfE), Ecodiseño, y Diseño para la Sustentabilidad (D4S) empezaron a ser utilizados.

El primero de estos calificativos, el Diseño para el Medio Ambiente, DfE, se originó en los EE.UU. en el año 1992 como un programa de la Agencia de Protección Ambiental de los Estados Unidos (EPA). El DfE trata de reducir el impacto global sobre la salud humana y el medio ambiente de un producto, proceso o servicio, donde los efectos son considerados a lo largo de todo su ciclo de vida. En este sentido dicho enfoque trata de aunar los dos grandes impulsos de la sociedad contemporánea: el desarrollo industrial y el desarrollo sostenible, minimizando los efectos del primero sobre el segundo, e incluyendo en este último aspecto la salud humana. Algunos prefieren denominar a este enfoque Diseño Verde, marcando un sesgo simplificador del abordaje con respecto a otros desarrollados posteriormente. (Encino Muñoz, 2014) Asimismo la noción de Ecodiseño surgió dentro de un manual elaborado en 1997 por el Programa de las Naciones Unidas para el Medio Ambiente - PNUMA (United Nations Environment Programme - UNEP en su siglas en inglés) junto con el Instituto Tecnológico de Delft, Holanda, cuya finalidad fue proporcionar una introducción al diseño ecológico para todos los interesados en el desarrollo de productos, tanto en empresas grandes como pequeñas, orientando a los involucrados en estas tareas y realizando reducciones radicales en el número de partes y en las cantidades de materiales utilizados, evitando, a su vez, el empleo de substancias tóxicas, con el objeto de disminuir y hacer eficiente el consumo de energía durante el uso del objeto diseñado, y finalmente, prever de manera planificada la posible reutilización, reciclaje o eliminación final del producto.

Por otra parte, el Diseño para la sustentabilidad, (D4S), de acuerdo a la definición proporcionada por el Programa de las Naciones Unidas para el Medio Ambiente - PNUMA, es un concepto más amplio que el ecodiseño o diseño para el medio ambiente, puesto que está vinculado con la relación sistema-producto (servicios sostenibles, innovación de sistemas y ciclo de vida de productos). Dicha noción es resultado de los ensayos tentativos anteriores y empezó a formularse hacia 2005. Es por ello que en la evaluación de los productos diseñados se van incorporando factores ambientales y sociales teniendo en cuenta la totalidad del ciclo de vida. Según este informe, la falta de conciencia en los países en vías de desarrollo respecto a esta temática constituye un gran obstáculo que es necesario superar. (Crul, 2007) De modo que, el D4S se sustenta en el análisis del ciclo de vida de un producto, el cual se inicia con la extracción, procesamiento y suministro de materias primas, la energía requerida para su producción, el transporte, el uso, considerando su posible reutilización y reciclaje, y su consecuente eliminación o final de vida, analizando los impactos ambientales que pudieran aparecer en cada etapa, lo cual constituye el modelo "de la cuna a la tumba". Cuestionando este enfoque, Gustavo Giuliano estima que "para algunos diseñadores no es medioambientalmente adecuado instrumentar políticas de producción en la línea señalada por las " 3 R" (reducir, reutilizar, reciclar) y la eco-eficiencia. Argumentan que si bien son conceptos aparentemente nobles, no señalan una estrategia de éxito 
a largo plazo en tanto no llegan a las raíces del problema". (Giuliano, 2014, págs. 80-81). La cuestión no reside en "hacer que las cosas incorrectas sean menos malas" sino en cambiar totalmente el enfoque, adoptando un nuevo paradigma que implique no pensar ya en controlar la naturaleza, sino aprender de ella respetándola, para lo cual hay que eliminar la idea de "residuo" y considerar el ciclo completo, esta vez, de la cuna a la cuna.

\section{Racionalidad limitada}

Para avanzar en nuestra argumentación debemos recuperar la noción de "racionalidad limitada" es decir, el reconocimiento de nuestra propia falibilidad en el momento de emitir juicios y realizar elecciones. Siguiendo a Herbert Simon:

1. la racionalidad exige un conocimiento y una anticipación completa de las consecuencias que seguirán a cada elección. En realidad, el conocimiento de las consecuencias es siempre fragmentario

2. dado que las consecuencias pertenecen al futuro, la imaginación debe suplir la falta de experiencia al asignarle valores. Pero sólo es posible anticipar de manera imperfecta los valores

3. la racionalidad exige una elección entre todos los posibles comportamientos alternativos. (Simon, 1972, pág. 76)

El pensamiento de Simon nos sirve no sólo porque se apoya en las "ciencias de lo artificial", es decir en la concepción de objetos artificiales o artefactos, sino, además porque supone una mirada basada en una actitud prospectiva. La prospectiva, es un tipo de anticipación y puede ser definida como un conjunto de metodologías o técnicas que son usadas con el objetivo de estudiar posibles escenarios futuros y su probabilidad, necesarias para tomar decisiones racionales, económicas o políticas.

Por otra parte, también es posible homologar el clásico concepto de proyecto con un punto de vista prospectivo. Recordemos que algunos definen "Proyectar" como el intento por conocer algo con anticipación: prever algo que sucederá. Mientras que otros consideran a "Proyectar" como el conjunto de acciones que se llevan a cabo en el presente para lograr que las cosas sucedan en el futuro. "Este segundo enfoque es más ajustado que el primero que sólo se limita a anticipar hechos. El proyecto hace que los hechos sucedan" (Cravino, 2005, pág. 103). Sin embargo, sabemos que muchas veces no es tan obvio llegar a un acuerdo con respecto a lo que es realmente sustentable ya que es difícil encontrar informaciones consensuadas o datos fehacientes que no generen controversia: desde las causas del calentamiento global o cambio climático, hasta el empleo o no de ciertos materiales y tecnologías. Solo bastaría ver algunos cuadros comparativos para comprobar la variabilidad de cifras. $Y$ esto nos lleva de nuevo al concepto de racionalidad limitada.

Es sabido que el análisis del ciclo de vida de un material incluye el estudio de las siguientes etapas: La extracción y manufactura de materias primas; El transporte a la planta de fabricación/montaje; Las actividades necesarias para el procesado de los materiales necesarios para la manufactura de los componentes; La distribución de los productos elaborados; El uso que se le da a los mismos; El reciclaje, refuncionalización y reempleo del producto una vez finalizado su uso inicial. Todas estas etapas requieren de la medición de variables, que como su nombre lo indica pueden fluctuar 
significativamente, puesto que no son iguales las formas de extracción o producción de un material, ni los kilómetros de transporte o la economía de los medios de traslado, ni tampoco los procedimientos de manufactura son necesariamente similares en todas partes del mundo. Asimismo, en los países más ricos la idea de "obsolescencia programada" y descarte está mucho más difundida que en otras regiones donde los objetos se reparan una y otra vez hasta, casi, extender ilimitadamente su vida útil.

Respecto, por ejemplo, al empleo de determinados materiales en el diseño de sillas se afirma que en el mundo occidental "entre 1960 y 1970 se inició una fase caracterizada por usar y tirar: la satisfacción de las necesidades a corto plazo y del placer hedonista". (Anderson, 2013)

Por el contrario, en la actualidad, respecto a la industria textil:

Muchas compañías también realizan diseños con materiales reciclables, con el fin de ayudar al medio ambiente; los residuos son un recurso muy valioso, pero a menudo poco comprendido. Recicladores de textiles han desarrollado una serie de prácticas socio-tecnológicas que permiten transformaciones de materiales, pero a menudo permanecen invisibles para las economías modernas". (López Barrios, 2012, pág. 78)

Es claro entonces que esta invisibilidad no permite recuperar y difundir experiencias valiosas. Sin embargo, respecto también a la producción textil, Francisco González Madariaga, a pesar de los exhaustivos cuadros que presenta concluye que

En cuanto a la evaluación de impacto ambiental, es muy difícil encontrar información de procesos y materiales especializados, por lo que realizar un correcto Análisis de Ciclo de Vida es costoso. Aunado a lo anterior, muchos de los productos de la industria del vestido contienen alta mezcla de materiales..." (González Madariaga, 2013, pág. 153).

En síntesis, para calificar un objeto artificial de sostenible se tiene que tener en cuenta determinados aspectos de los que no se tiene demasiada información o se sospecha de la veracidad de la misma, lo cual limita la toma de decisiones y la racionalidad de estas:

1) Materiales y recursos empleados, debiéndose tener preferencia por aquellos naturales, reciclables, reciclados y más duraderos.

No obstante esta decisión es dificultosa pues debería contarse con "eco-etiquetas" que certifiquen de manera comprobable, por ejemplo, que una madera proviene de un bosque implantado y no de una reserva protegida. Igualmente, respecto a la elección entre PVC (Cloruro de polivinilo) que contiene cloro, substancia tóxica y cancerígena, y otros plásticos como polipropileno o polietileno de alta densidad, se debe tener en cuenta que el primero tiene una duración muy superior a los otros dos. En este sentido, vale reconocer que existe una amplia literatura tanto en contra (Greenpeace, 2002), como a favor del PVc (Europeas, 2000), lo cual no contribuye a facilitar ninguna decisión. 
Es interesante, asimismo, algunas experiencias que, desde lo académico, empiezan a investigar de manera interdisciplinaria sobre "nuevos" materiales, es decir, sobre el empleo de aquello que habitualmente se descartaba. (García Solano, Barajas Sepúlveda, \& Alarcón Aranguren, 2014)

2) Energía consumida durante la extracción, transporte, fabricación, uso y reciclado de un producto.

María del Rosario Bernatene y Guillermo Juan Canale sostienen que "las herramientas para hacer Análisis de Ciclo de Vida juegan un rol cada vez más importante en la comunicación entre las empresas manufactureras y sus partes interesadas (stakeholders), como por ejemplo en el eco-etiquetado o procesos de compras verdes." (Bernatene \& Canale, 2018, pág. 157). No obstante, también consideran que se hace dificultoso integrar a un mismo tiempo los aspectos ambientales, de la calidad y de costos cuando los resultados de un ACV sólo se aplican a la mejora de un aspecto acotado de un producto. Además, aseguran que

aunque están pautados por normativa bastante precisa, los análisis de Ciclo de Vida exhaustivos, a como los definen la ISO 14040 y 14044 , son caros, toman considerable tiempo para poder recolectar toda la información y procesarla de manera consistente y aun así, ciertos parámetros son inciertos (Bernatene \& Canale, 2018, pág. 157).

Energía incorporada por distintos materiales de construcción, según diversos autores. La energía está expresada en $\mathrm{kWh} / \mathrm{kg}$ de producto, salvo que se indique otra cosa.
Tabla 1. Fuente (Vázquez Espí, 2001, págs. 34-35).

\begin{tabular}{|c|c|c|c|c|c|c|c|c|}
\hline \multirow[b]{2}{*}{ Materiales } & \multicolumn{8}{|c|}{ Referencias (año) } \\
\hline & $1-1997$ & $2-1982$ & $3-1978$ (b) & 4-1995 & $5-1998$ & 61979 (c) & $7-2000(e)$ & $8-1990$ \\
\hline Acero & 8,06 & 10 & 7,67 & 7-13 & 7-11 & 13 & 11 & $14(g)$ \\
\hline Aluminio & 27,0 & & & $42-61$ & $42-67$ & 73 & $44-60$ & $81(g)$ \\
\hline Cemento & & 2,2 & 1,8 & & & 2,4 & 2 & \\
\hline Hormigón & 0,28 & 0,2 & 0,5 & 0,2 & & 0,3 & 0,7 & \\
\hline Ladrillo cerámico & 0,86 & 1,2 & & $0,7-1,69$ & & 0,09 & 1,25 & \\
\hline Madera & & 0,1 & & 1,25 & & & & \\
\hline Plástico & 45 & 10 & 2,73 & $22-61$ & & 2,65 & $20-40$ & $21-23$ \\
\hline Vidrio & 9,19 & 6,0 & & $3,6-7$ & $3,3-8,3$ & 7,4 & 5,3 & $22,5(f)$ \\
\hline
\end{tabular}

Por otra parte, como vemos en el cuadro precedente, no es posible transpolar las evaluaciones de un mismo material realizado en un determinado lugar y tiempo y aplicarlas en otro contexto. Sin embargo, muchas veces es más fácil obtener información contrastable y fehaciente de lo que gasta un determinado producto durante su vida útil que la energía consumida previamente. 
3) Gestión de residuos: Este es uno de los puntos más oscuros, puesto que en este aspecto están involucrados los estados nacionales que, si bien pretenden tener políticas amigables con el medio ambiente, tienden a proteger industrias claves en la economía de cada país. Obviamente, en la medida en que las normativas difieren entre un lugar y otro, lo que es lícito y no, y lo que se afirma o se oculta varía (Puig Ventosa, 2007). En este sentido, es oportuno evaluar los modelos que proponen analizar desde variables cuantitativas a los residuos de construcción (Mercader-Moyano, Ramírez de Arellano-Agudo, \& Ruesga-Díaz, 2017).

4) Salud: Similar al punto anterior es el aspecto de si determinados productos emiten substancias nocivas para la salud o afectan al medio ambiente, incluyendo también elementos peligrosos o dañinos para la seguridad, la durabilidad o el uso de artefactos o lugares. Vale también destacar como en el caso del diseño de indumentaria los textiles naturales ganaron reconocimiento y se empezó a cuestionar el uso de determinadas sustancias químicas (Musso, 2012). Empero, en este sentido también algunos autores señalan que todavía las empresas, ni los clientes potenciales, consideran como una variable de competitividad tener, por ejemplo, producciones de bajas emisiones (Castellanos Alvarado \& Fernández Marín, 2011).

5) Uso de un objeto de diseño: Es necesario en este ítem replantearnos qué entendemos por uso de un artefacto, ya que, como hemos mencionado y como se puede corroborar con respecto a la durabilidad, por ejemplo, de los automóviles en los países más pobres del planeta, un objeto de diseño industrial puede extender su vida útil, con pequeñas tareas de mantenimiento, más allá de los límites planteados inicialmente, aunque queda como reflexión analizar si este uso ampliado no genera consecuencias indeseadas: polución, contaminación visual y sonora, ineficiencia, entre otros.(Valenciana, 2010)

6) Reciclaje, reutilización y reducción: Tampoco hay información generalizable y común a todos más allá de las buenas intenciones. Sostiene Daniela Edurne Battista que el diseño sustentable aunque tiene una raíz ecológica, su introducción en Argentina se vio ligada a las crisis económicas, apareciendo como paliativo y en consecuencia asociada a ciertas prácticas como la reutilización de materiales, entre otros. “En ese sentido puede entenderse al diseño sustentable en Argentina como resultado de una situación económico-social antes que una concientización sobre las problemáticas ambientales." (Edurne Battista, 2012, pág. 1)Vale destacar que lo que en sociedad basadas en el consumismo, el reciclaje y reutilización resulta un desafío y una excepción, mientras que en otras, habituadas a la carencia, es una práctica cotidiana, y todo, pero prácticamente todo, puede ser útil para alguien que acepte esa oportunidad.

\section{Forma "sustentable"}

La forma, ya hemos dicho en alguna oportunidad, tiene un telos o principio de acción, pero la materia, conceptualmente pura, no. Sin embargo los materiales empleados "al exigir una interpretación correcta sobre sus posibilidades de uso tienden a establecer o estimular criterios valorativos sobre el modo en que se emplean, determinando posiciones conceptuales sobre la percepción correcta o incorrecta, verdadera o falsa, de su expresión." (Cravino \& Pokropek, 2018, 

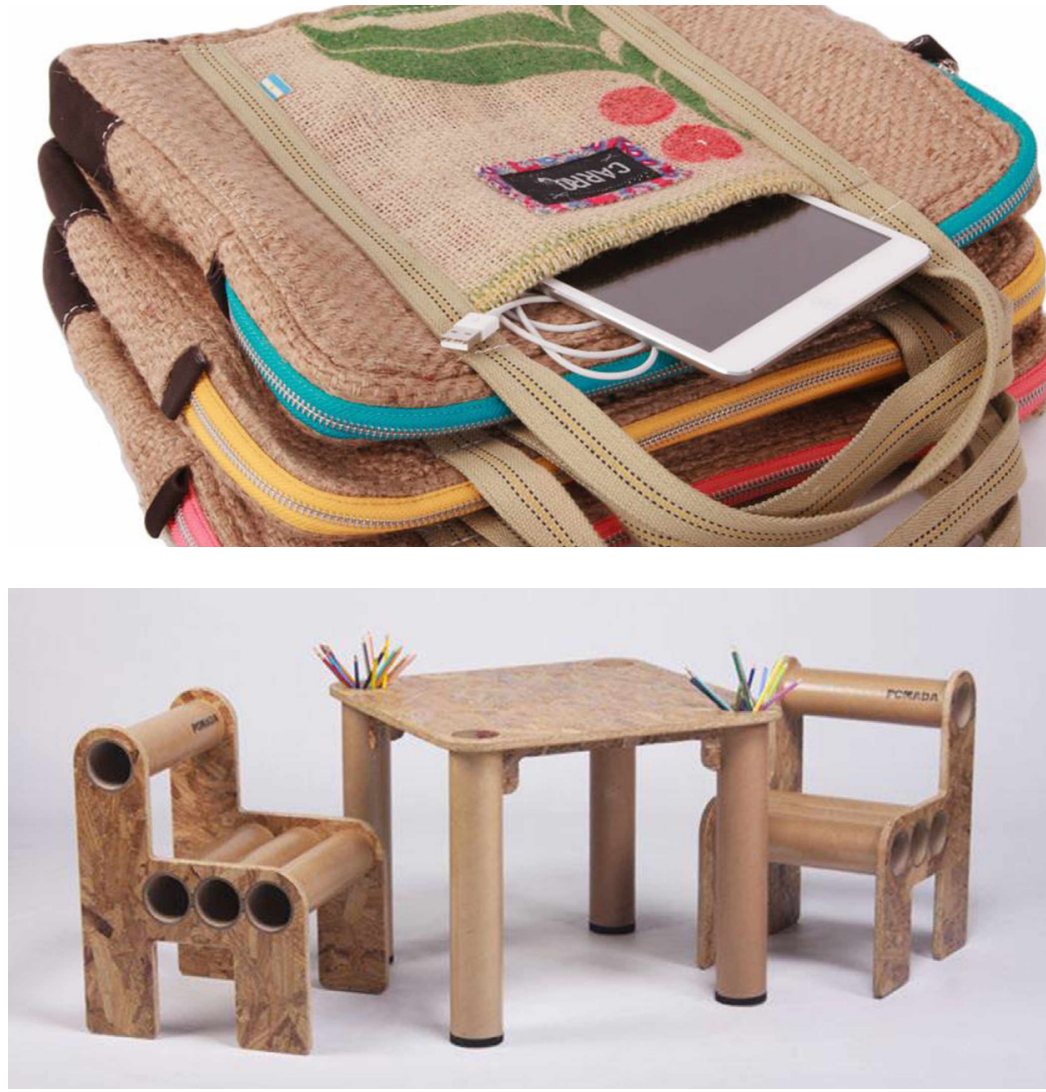

Figura 1. Bolsos realizados con bolsas de arpillera y telas de descarte. Carro, Diseñadoras Silvina Cannito y Guillermina Balsells.

Figura 2. Muebles para niños realizados con tubos de cartón descartados por la industria gráfica. Pomada, Diseñadores: Antonela Dada y Bruno Sala Conill. América Latina Diseña Sustentable (Buenos Aires, 2014)

pág. 17). Esta expresión de la materia es un vehículo que comunica valores, entre ellos, los ligados al cuidado del medio ambiente.

Al no contar con datos fehacientes, resulta arduo para los diseñadores decidir respecto a la sustentabilidad de los materiales empleados, ya que la información se encuentra fragmentada o es de dudosa fuente. Y por ende, la resolución respecto a cómo usarlos también se ve afectada. Asimismo, no es posible suponer comportamientos futuros de productos y materiales en base a testimonios inciertos. La economía muchas veces fija el rumbo de la ecología, y sobre la base de la primera se toman decisiones que afectan a la segunda. Una publicación realizada conjuntamente entre la Ciudad de Buenos Aires y México DF denominada América Latina Diseña Sustentable pone en evidencia el trabajo de diseñadores y artesanos que afectados por diversas circunstancias, fundamentalmente económicas, adoptan como recurso viable trabajar con materiales reciclados. Estos materiales, que en otras partes del mundo hubiesen sido calificados como desecho o basura, y terminarían siendo motivo de estudio respecto a cómo hacer para eliminarlos, en América Latina se transforman en recurso valioso y en oportunidad de innovación. Materiales cuyo ciclo de vida no es, aún, motivo de rigurosos estudios. Por eso es indispensable el cambio de mirada. Sin embargo, vale destacar que no es el material en sí lo que le otorga valor a lo producido, sino la calidad del diseño que aprovecha el recurso, que transforma la escasez en oportunidad. Es por ello que en dicha publicación, una de las diseñadoras que exhibe sus productos, sostiene que "Nos gusta aprovechar los materiales y tomamos el desafío de reutilizar descartes industriales. Si bien no es una tarea sencilla, hay que estar 
atentos porque en cualquier lugar puede aparecer la materia prima óptima" (Gobierno de la Ciudad Autónoma de Buenos Aires, 2014, pág. 17).

Semejante apreciación tiene otro grupo de diseñadores que sostienen que

Recurrimos al descarte y al reciclado por tres factores que para nosotros son bien claros: porque es un material que tenemos disponible, porque contamos con el capital humano o con las herramientas necesarias para manejarlo, y porque no tenemos el capital monetario para incorporar otras opciones" (Buenos Aires, 2014)

Ecología y economía van de la mano y no son alternativas contrapuestas.

\section{Conclusiones}

Las dificultades referidas que se presentan al diseñar de manera sustentablemente consciente hace se tomen decisiones relativas a la forma del objeto que puedan manifestar de manera explícita el compromiso ambiental asumido. Esto hace que el diseño sustentable permanezca vinculado al diseño de autor con una impronta artesanal y con una escala acotada de producción muchas veces, pero también relacionado con economías basadas en el desarrollo local o en la producción de micro emprendedores. Economías que se nutren de manera novedosa de aquello que otros han descartado. Es por ello que podemos hablar de "Ecodiseño" y no plenamente de un "Diseño para la sustentabilidad", puesto que no es posible estimar las consecuencias que el empleo de ciertos recursos y materiales tengan para el medio ambiente en la medida que la racionalidad es limitada y la información al respecto sea fragmentaria. Y en este sentido es también importante reconocer el diálogo fecundo y el mutuo aprendizaje que aparece entre artesanos y diseñadores, cuyas sensibilidades comunes permiten suplir las dificultades que surgen de los efectos de esta "racionalidad limitada". Sensibilidades que muchas veces recuperan saberes ancestrales en armonía con el medio ambiente. De modo que estas circunstancias, definidas muchas veces por la carencia y la austeridad, permiten adoptar un enfoque sustentable, definido necesariamente desde lo local, donde se preste atención a la diversidad sociocultural y ambiental.

De modo que, supliendo la falta de información fehaciente respecto, por ejemplo, al ciclo de vida de los materiales empleados, lo sustentable se define entonces por la apariencia material del producto elaborado y por su explícita utilización de aquello que la sociedad descarta, dándole, por medio del diseño, una significativa y renovada apariencia.

\section{Referencias}

Anderson, I. (2013). La estética burguesa en el diseño de sillas. Arte e Investigación (9), 20-25.

Astier, M., Masera, O. R., \& Galván-Miyoshi, Y. (2008). Evaluación de sustentabilidad. Un enfoque dinámico y multidimensional. Valencia, España: El Colegio de la Frontera Sur. Universidad Nacional Autónoma de México. Centro de Investigaciones en Ecosistemas. Bernatene, M. d.-C. (2018). Innovación sustentable en Diseño a partir de la integración del análisis de Ciclo de Vida (ACV) con Cadenas Globales de Valor (CGV).
Cuadernos del Centro de Estudios en Diseño y Comunicación. Ensayos (69), 151-174.

Buenos Aires, C. (2014). América Latina diseña sustentable. Buenos Aires, México DF: Gobierno de la Ciudad de Buenos Aires, Gobierno de México DF.

Cangi, A. (2015). Cuadro Ecológico. Cartografías del Sur. Revista Multidisciplinaria en Ciencias, Arte y Tecnología de la Universidad Nacional de Avellaneda , 81-91.

Capra, F. (2003). La trama de la vida. Barcelona: Anagrama. 
Castellanos Alvarado, C., \& Fernández Marín, L. (2011). Hacia la sostenibilidad. Ideas de negocios ecoeficientes. 4 to Congreso Internacional de Diseño (págs. 108-115). México DF: Universidad Iberoamericana, México.

CEPAL. (1971). El medio ambiente humano y el desarrollo económico en América Latina. Santiago de Chile: Naciones Unidas.

Cravino, A. (2005). Reflexiones sobre la Teoría y la Crítica. Buenos Aires: Editorial Praia.

Cravino, A., \& Pokropek, J. (2018). Algunas precisiones sobre la borrosa noción de Materia para el Diseño Interior. Cuadernos del Centro de Estudios en Diseño y Comunicación. Ensayos (70), 15-27.

Crul, M. -D. (2007). Diseño para la Sostenibilidad. Un enfoque práctico para economías. Delft: Programa de Naciones Unidas para el Medio Ambiente, PNUMA.

D’Amico, F. C. (2000). Arquitectura Bioclimática, Conceptos Básicosy Panorama Actual. Madrid: Instituto Juan De Herrera.

Edurne Battista, D. (2012). Sustentabilidad en el diseño en Argentina. VI Jornadas de Investigación en Disciplinas Artísticas y Proyectuales (pág. 10). La Plata: Facultad de Bellas Artes, Universidad Nacional de la Plata.

Encino Muñoz, A. G. (diciembre de 2014). El diseñador industrial y la producción de mobiliario: una perspectiva desde la. Entreciencias: diálogos en la Sociedad del Conocimiento , 263-275.

Europeas, C. d. (2000). Libro Verde: Cuestiones medioambientales relacionadas con el PVC. Bruselas.

Evans, M., \& De Schiller, S. (1988). Diseño bioambientaly arquitectura solar. Buenos Aires: EUDEBA.

García Solano, H., Barajas Sepúlveda, D. F., \& Alarcón Aranguren, L. M. (2014). Experimentación de materiales, un camino para la sustentabilidad en el diseño. Revista de Investigación, Desarrollo e Innovación, 4 (2), 87-94.

Giglo, N. (2006). Estilos de desarrollo y medio ambiente en América Latina, un cuarto de siglo después. Santiago de Chile: CEPAL.

Giuliano, G. (2014). De la cuna a la cuna: una crítica al diseño ecoeficiente Artículo publicado en Vol. III, 2014. Revista Argentina de Ingeniería , III, 76-83.

Gobierno de la Ciudad Autónoma de Buenos Aires. (2014). Carro. En G. d. Aires, América Latina Diseña Sustentable (págs. 14-18). Buenos Aires: Buenos Aires Ciudad.

González Madariaga, F. J. (2013). Ecoeficiencia. Propuesta de diseño para el Mejoramiento ambiental. Guadalajara:
Editorial Universitaria, Centro Universitario de Arte, Arquitectura y Diseño. Universidad de Guadalajara.

Greenpeace. (2002). Hacia un futuro libre de PVC: Restricciones gubernamentales y empresariales al vinilo. Raleigh, Carolina del Norte, EEUU: Greenpeace Internacional. Herrera, A. (1977). ¿Catástrofe o Nueva Sociedad?. Modelo Mundial Latinoamericano. San Carlos de Bariloche: CIID. Fundación Bariloche.

Iussa, E., Granero, V., Govoni, P., Argüello, R., \& Gatani, M. (2015). Compuestos aglomerados a base de cáscaras de maní en matriz polimérica. Una aproximación al análisis de ciclo de vida. En R. T. Bongiovanni, Avances y Estado de situación en Análisis de Ciclo de vida y Huellas ambientales en Argentina (págs. 47-49). Córdoba: Instituto Nacional de Tecnología Industrial, Regional Córdoba.

López Barrios, M. C. (2012). El impacto ambiental del fast fashion pronta moda. Arquetipos (4), 72-8o.

Meadows, D. (1982). Los límites del crecimiento. México: Fondo de Cultura Económica.

Mercader-Moyano, P., Ramírez de Arellano-Agudo, A. C.-C., \& Ruesga-Díaz, D. (diciembre de 2017). Sistema BIM de cuantificación automática de los residuos de construcción y demolición. Método de transferencias ponderadas de la medición. "Estudios del hábitat , 1-9".

Musso, M. L. (2012). Ecología y color en textiles desde Ios noventa hasta la actualidad. AREA - Agenda de Reflexión en Arquitectura, Diseño y Urbanismo, 65-75.

Olgyay, V. (1998). Arquitectura y clima - Manual de diseño bioclimático para arquitectos y urbanistas. Barcelona: Gustavo Gili.

Puig Ventosa, I. (2007). Fiscalidad verde y residuos. (A. y. Instituto Sindical de Trabajo, Ed.) Daphnia, 17-18.

Simon, H. (1972). El comportamiento administrativo. Madrid: Aguilar. Sunkel, O., \& Gligo, N. (1980). Estilos de desarrollo y medio ambiente en la América Latina. México: Fondo de Cultura Económica.

Valenciana, I. d. (2010). El ecodiseño en Europa, estado, situación y tendencia. Valencia: Cámara de Valencia.

Vázquez Espí, M. (2001). Construcción e impacto sobre el ambiente: el caso de la tierra y otros materiales. Informes de la Construcción, 52 (471), 29-43.

Vilchez, A., Gil Pérez, D., Toscano, J., \& Macías, O. (2010). Década de la educación para la sostenibilidad. Madrid: Centro de Altos Estudios Universitarios de la OEI. 